\title{
Ideal Statistically Pre-Cauchy Triple Sequences of Fuzzy Number and Orlicz Functions
}

\author{
Xue Feng \\ College of Mathematics and Statistics, Qinghai Minzu University, Xining, China \\ Email: fengx8023@163.com
}

How to cite this paper: Feng, X. (2021) Ideal Statistically Pre-Cauchy Triple Sequences of Fuzzy Number and Orlicz Functions. Applied Mathematics, 12, 767-774. https://doi.org/10.4236/am.2021.129051

Received: July 28, 2021

Accepted: August 31, 2021

Published: September 3, 2021

Copyright $\odot 2021$ by author(s) and Scientific Research Publishing Inc. This work is licensed under the Creative Commons Attribution International License (CC BY 4.0).

http://creativecommons.org/licenses/by/4.0/

\section{Abstract}

In this paper, we extend the notions of ideal statistically convergence for sequence of fuzzy number. We introduce the notions ideal statistically preCauchy triple sequences of fuzzy number about Orlicz function, and give some correlation theorem. It is shown that $x=\left\{x_{i j k}\right\}$ is ideal statistically preCauchy if and only if $\left\{(m, n, t) \in N \times N \times N: \frac{1}{m^{2} n^{2} t^{2}} \mid\{(i, j, k)\right.$ : $\left.\left.D\left(x_{i j k}, x_{p q r}\right) \geq \varepsilon, i \leq m, j \leq n, t \leq k\right\} \mid \geq \delta\right\} \in I$. At the same time, we have proved $x=\left\{x_{i j k}\right\}$ is ideal statistically convergent to $x_{0}$ if and only if $\left\{(m, n, t) \in N \times N \times N: \frac{1}{m n t} \sum_{i=1}^{m} \sum_{j=1}^{n} \sum_{k=1}^{t} M\left(\frac{D\left(x_{i j k}, x_{0}\right)}{\rho}\right) \geq \delta\right\} \in I$. Also, some properties of these new sequence spaces are investigated.

\section{Keywords}

Fuzzy Numbers, Ideal Statistical Convergence, Orlicz Functions

\section{Introduction}

The notion of statistical convergence was introduced by Fast [1] and also independently by Buck [2] and Schoenberg [3] for real and complex sequences.Over the years and under different names statistical convergence has been discussed in the theory of Fourier analysis, Ergodic theory and Number theory. Later on it was further investigated from the sequence spaces point of view and linked with summability theory by Altinok and Et [4], Connor [5], Et et al. ([6] [7] [8]), Fridy [9], Fridy and Orhan [10], Mursaleen [11] and many others.

Matloka [12] defined the notion of fuzzy sequence and introduced bounded 
and convergent sequences of fuzzy real numbers and studied their some properties. After then, Nuray and Savas [13] defined the notion of statistical convergence for sequences of fuzzy numbers. Since then, there has been increasing interest in the study of statistical convergence of fuzzy sequences (see [14]-[19]).

Lindesstrauss and Tzafriri [20] used the idea of Orlicz sequence space, $l_{M}:=\left\{x \in \omega: \sum_{k=1}^{\infty} M\left(\frac{|x|}{\rho}\right)<\infty\right.$, for some $\left.\rho>0\right\}$, which is Banachi space with the norm: $\|x\|_{M}=\inf \left\{\rho>0: \sum_{k=1}^{\infty} M\left(\frac{|x|}{\rho}\right) \leq 1\right\}$. The space $l_{M}$ is closely related to the space $l_{p}$, which is an Orlicz sequence space with $M(x)=x^{p}$ for $1 \leq p<\infty$.

Connor, Fridy and Kline [21] proved that statistical convergent sequences are statistically pre-Cauchy and any bounded statistically pre-Cauchy sequence with nowhere dense set of limit points is statistically convergent. They also gave an example showing statistically pre-Cauchy sequences are not necessarily statistically convergent.

In this paper, we extend the notions of ideal statistically convergence for sequence of fuzzy number. We introduce the notions ideal statistically pre-Cauchy triple sequences of fuzzy number about Orlicz function, and give some correlation theorem. Also, some properties of these new sequence spaces are investigated. It popularized the work of predecessors.

\section{Definitions and Preliminaries}

In this section, we give some basic notions which will be used throughout the paper.

Let $\tilde{A} \in \tilde{F}(R)$ be a fuzzy subset on $R$. If $\tilde{A}$ is convex, normal, upper semicontinuous and has compact support, we say that $\tilde{A}$ is a fuzzy number. Let $\tilde{R}^{c}$ denote the set of all fuzzy numbers.

For $\tilde{A} \in \tilde{R}^{c}$, we write the level set of $\tilde{A}$ as $A_{\lambda}=\{x: A(x) \geq \lambda\}$ and $A_{\lambda}=\left[A_{\lambda}^{-}, A_{\lambda}^{+}\right]$. Let $\tilde{A}, \tilde{B} \in \tilde{R}^{c}$, we define $\tilde{A}+\tilde{B}=\tilde{C}$ iff $A_{\lambda}+B_{\lambda}=C_{\lambda}$, $\lambda \in[0,1]$ iff $A_{\lambda}^{-}+B_{\lambda}^{-}=C_{\lambda}^{-}$and $A_{\lambda}^{+}+B_{\lambda}^{+}=C_{\lambda}^{+}$for any $\lambda \in[0,1] . A_{\lambda} \cdot B_{\lambda}=C_{\lambda}$, where

$$
\begin{aligned}
& C_{\lambda}^{-}=\min \left\{A_{\lambda}^{-} \cdot B_{\lambda}^{-}, A_{\lambda}^{-} \cdot B_{\lambda}^{+}, A_{\lambda}^{+} \cdot B_{\lambda}^{-}, A_{\lambda}^{+} \cdot B_{\lambda}^{+}\right\}, \\
& C_{\lambda}^{+}=\max \left\{A_{\lambda}^{-} \cdot B_{\lambda}^{-}, A_{\lambda}^{-} \cdot B_{\lambda}^{+}, A_{\lambda}^{+} \cdot B_{\lambda}^{-}, A_{\lambda}^{+} \cdot B_{\lambda}^{+}\right\} .
\end{aligned}
$$

Define

$$
D(\tilde{A}, \tilde{B})=\sup _{\lambda \in[0,1]} d\left(A_{\lambda}, B_{\lambda}\right)=\sup _{\lambda \in[0,1]} \max \left\{\left|A_{\lambda}^{-}-B_{\lambda}^{-}\right|,\left|A_{\lambda}^{+}-B_{\lambda}^{+}\right|\right\},
$$

where $d$ is the Hausdorff metric. $D(\tilde{A}, \tilde{B})$ is called the distance between $\tilde{A}$ and $\tilde{B}$.

Using the results of [22] [23], we see that

1) $\left(\tilde{R}^{c}, D\right)$ is a complete metric space,

2) $D(u+w, v+w)=D(u, v)$, 
3) $D(k u, k v)=|k| D(u, v), k \in R$,

4) $D(u+v, w+e) \leq D(u, w)+D(v, e)$,

5) $D(u+v, \overline{0}) \leq D(u, \overline{0})+D(v, \overline{0})$,

6) $D(u+v, w) \leq D(u, w)+D(v+\overline{0})$,

Where $u, v, w, e \in \tilde{R}^{c}, \tilde{0}$ represents zero fuzzy number.

A sequence $\left\{x_{n}\right\}$ of fuzzy numbers is said to be statistically convergent to a fuzzy number $x_{0}$ if for each $\varepsilon>0$ the set $A(\varepsilon)=\left\{n \in N: D\left(x_{n}, x_{0}\right) \geq \varepsilon\right\}$ has natural density zero. The fuzzy number $x_{0}$ is called the statistical limit of the sequence $\left\{x_{n}\right\}$ and we write $s t-\lim _{n \rightarrow \infty} x_{n}=x_{0}$ [24].

The concept of Orlicz function was introduced by Parashar and Choudhary [25], A mapping $M:[0, \infty) \rightarrow[0, \infty)$ is said to be an Orlicz funtion [26]

1) $M(0)=0$ iff $x=0$,

2) $M(x)>0$ for $x>0$,

3) $M(x) \rightarrow \infty$ as $x \rightarrow \infty$,

4) $M$ is continuous, nondecreasing and convex.

An Orlicz function may be bounded or unbounded. For example, $M(x)=x^{p}(0<p \leq 1)$ is bounded.

A triple sequence can be defined as a function $X: N \times N \times N \rightarrow R(C)$ where $N, R$ nad $C$ denote the set of natural numbers, real numbers and complec numbers respectively. A triple sequence $\left\{x_{i j k}\right\}$ is said to be Cauchy sequence if for every $\varepsilon>0$, there exist $N(\varepsilon) \in N$ such that $\left|x_{i j k}-x_{p q r}\right|<\varepsilon$ whenever $i, p \geq N, \quad j, q \geq N, \quad k, r \geq N \quad$ [27].

A triple sequence $\left\{x_{i j k}\right\}$ is called statistically pre-Cauchy if for every $\varepsilon>0$ there exist $p=p(\varepsilon), q(\varepsilon)$ and $r(\varepsilon)$ such that

$$
\lim _{m, n, t \rightarrow \infty}\left|\frac{1}{m^{2} n^{2} t^{2}}\right| x_{i j k}-x_{p q r}|\geq \varepsilon, i \leq m, j \leq n, t \leq k|=0 .
$$

where the vertical bars indicate the number of elements in the set [28].

\section{Main Results}

Definition 3.1. A triple sequence of fuzzy numbers is said to be ideal statistically pre-Cauchy if for every $\varepsilon>0, \delta>0$ there exist $p=p(\varepsilon), q(\varepsilon)$ and $r(\varepsilon)$ such that

$$
\left\{(m, n, t) \in N \times N \times N: \frac{1}{m^{2} n^{2} t^{2}}\left|\left\{(i, j, k): D\left(x_{i j k}, x_{p q r}\right) \geq \varepsilon, i \leq m, j \leq n, t \leq k\right\}\right| \geq \delta\right\} \in I .
$$

where the I denote the nontrivival ideal of $N$.

Theorem 3.1. Let $x=\left\{x_{i j k}\right\}$ be a triple sequence of fuzzy number and let $M$ be a bounded Oricz function. Then $x$ is ideal statistically pre-Cauchy if and only if

$$
\left\{(m, n, t) \in N \times N \times N: \frac{1}{m^{2} n^{2} t^{2}} \sum_{i, p \leq m} \sum_{j, q \leq n} \sum_{k, r \leq t} M\left(\frac{D\left(x_{i j k}, x_{p q r}\right)}{\rho}\right) \geq \delta\right\} \in I .
$$

Proof. Suppose that 


$$
\left\{(m, n, t) \in N \times N \times N: \frac{1}{m^{2} n^{2} t^{2}} \sum_{i, p \leq m} \sum_{j, q \leq n} \sum_{k, r \leq t} M\left(\frac{D\left(x_{i j k}, x_{p q r}\right)}{\rho}\right) \geq \delta\right\} \in I .
$$

For each $\varepsilon>0, \delta>0$ and $\rho>0, m, n, t \in N$, we have

$$
\begin{aligned}
& \frac{1}{m^{2} n^{2} t^{2}} \sum_{i, p \leq m} \sum_{j, q \leq n} \sum_{k, r \leq t} M\left(\frac{D\left(x_{i j k}, x_{p q r}\right)}{\rho}\right) \\
= & \frac{1}{m^{2} n^{2} t^{2}} \sum_{i, p \leq m} \sum_{j, q \leq n} \sum_{k, r \leq t D\left(x_{i j k}, x_{p q r}\right) \leq \varepsilon} M\left(\frac{D\left(x_{i j k}, x_{p q r}\right)}{\rho}\right) \\
& +\frac{1}{m^{2} n^{2} t^{2}} \sum_{i, p \leq m} \sum_{j, q \leq n} \sum_{k, r \leq t D\left(x_{i j k}, x_{p q r}\right) \geq \varepsilon} M\left(\frac{D\left(x_{i j k}, x_{p q r}\right)}{\rho}\right) \\
\geq & \frac{1}{m^{2} n^{2} t^{2}} \sum_{i, p \leq m} \sum_{j, q \leq n} \sum_{k, r \leq t D\left(x_{j i k}, x_{p q r}\right) \leq \varepsilon} M\left(\frac{D\left(x_{i j k}, x_{p q r}\right)}{\rho}\right) \\
\geq & M(\varepsilon)\left\{\frac{1}{m^{2} n^{2} t^{2}}\left\{(i, j, k): D\left(x_{i j k}, x_{p q r}\right) \geq \varepsilon, i \leq m, j \leq n, k \leq t\right\} \mid \geq \delta\right\} \in I .
\end{aligned}
$$

Now suppose that $x$ is ideal statistically pre-Cauchy and that $\varepsilon$ has been given.

Let $\varepsilon>0, \delta>0$ be such that $M(\xi)<\frac{\varepsilon}{2}$.

Since $M$ is bounded Orlicz function, there exist an integer $G$ such that $M(x)<\frac{G}{2}$ for all $x \geq 0$.

Not that, for each $m, n, t \in N$

$$
\begin{aligned}
& \frac{1}{m^{2} n^{2} t^{2}} \sum_{i, p \leq m} \sum_{j, q \leq n} \sum_{k, r \leq t} M\left(\frac{D\left(x_{i j k}, x_{p q r}\right)}{\rho}\right) \\
= & \frac{1}{m^{2} n^{2} t^{2}} \sum_{i, p \leq m} \sum_{j, q \leq n} \sum_{k, r \leq t D\left(x_{i j k}, x_{p q r}\right)<\xi} M\left(\frac{D\left(x_{i j k}, x_{p q r}\right)}{\rho}\right) \\
& +\frac{1}{m^{2} n^{2} t^{2}} \sum_{i, p \leq m} \sum_{j, q \leq n} \sum_{k, r \leq t D\left(x_{i j k}, x_{p q r}\right) \geq \xi} M\left(\frac{D\left(x_{i j k}, x_{p q r}\right)}{\rho}\right) \\
\leq & \left.M(\xi)+\frac{1}{m^{2} n^{2} t^{2}} \sum_{i, p \leq m} \sum_{j, q \leq n} \sum_{k, r \leq t D\left(x_{j i k}, x_{p q r}\right)}\right) \geq \xi\left(\frac{D\left(x_{i j k}, x_{p q r}\right)}{\rho}\right) \\
\leq & \frac{\varepsilon}{2}+\frac{G}{2}\left\{\frac{1}{m^{2} n^{2} t^{2}}\left|\left\{(i, j, k): D\left(x_{i j k}, x_{p q r}\right) \geq \xi, i \leq m, j \leq n, k \leq t\right\}\right| \geq \delta\right\} \\
\leq & +G\left\{\frac{1}{m^{2} n^{2} t^{2}}\left|\left\{(i, j, k): D\left(x_{i j k}, x_{p q r}\right) \geq \xi, i \leq m, j \leq n, k \leq t\right\}\right| \geq \delta\right\} \in I .
\end{aligned}
$$

Hence

$$
\left\{(m, n, t) \in N \times N \times N: \frac{1}{m^{2} n^{2} t^{2}} \sum_{i, p \leq m} \sum_{j, q \leq n} \sum_{k, r \leq t} M\left(\frac{D\left(x_{i j k}, x_{p q r}\right)}{\rho}\right) \geq \delta\right\} \in I .
$$

Theorem 3.2. Let $x=\left\{x_{i j k}\right\}$ be a triple sequence of fuzzy numbers and let $M$ be a bounded Orlicz function. Then $x$ is ideal statistically convergent to $x_{0}$ if 
and only if

$$
\left\{(m, n, t) \in N \times N \times N: \frac{1}{m n t} \sum_{i=1}^{m} \sum_{j=1}^{n} \sum_{k=1}^{t} M\left(\frac{D\left(x_{i j k}, x_{0}\right)}{\rho}\right) \geq \delta\right\} \in I .
$$

Proof. Suppose that

$$
\left\{(m, n, t) \in N \times N \times N: \frac{1}{m n t} \sum_{i=1}^{m} \sum_{j=1}^{n} \sum_{k=1}^{t} M\left(\frac{D\left(x_{i j k}, x_{0}\right)}{\rho}\right) \geq \delta\right\} \in I .
$$

For each $\varepsilon>0, \delta>0$ and $\rho>0, m, n, t \in N$, we have

$$
\begin{aligned}
& \frac{1}{m n t} \sum_{i=1}^{m} \sum_{j=1}^{n} \sum_{k=1}^{t} M\left(\frac{D\left(x_{i j k}, x_{0}\right)}{\rho}\right) \\
& =\frac{1}{m n t} \sum_{i=1}^{m} \sum_{j=1}^{n} \sum_{k=1, D\left(x_{i j k}, x_{0}\right) \geq \varepsilon}^{t} M\left(\frac{D\left(x_{i j k}, x_{0}\right)}{\rho}\right) \\
& \quad+\frac{1}{m n t} \sum_{i=1}^{m} \sum_{j=1}^{n} \sum_{k=1, D\left(x_{i j k}, x_{0}\right)<\varepsilon}^{t} M\left(\frac{D\left(x_{i j k}, x_{0}\right)}{\rho}\right) \\
& \geq \frac{1}{m n t} \sum_{i=1}^{m} \sum_{j=1}^{n} \sum_{k=1, D\left(x_{i j k}, x_{0}\right) \geq \varepsilon}^{t} M\left(\frac{D\left(x_{i j k}, x_{0}\right)}{\rho}\right) \\
& \geq M(\varepsilon)\left\{\frac{1}{m n t}\left|\left\{(i, j, k): D\left(x_{i j k}, x_{0}\right) \geq \varepsilon, i \leq m, j \leq n, k \leq t\right\}\right| \geq \delta\right\} \in I .
\end{aligned}
$$

We have $x$ is ideal statistically convergent to $x_{0}$.

Now suppose that $x$ is ideal statistically convergent to $x_{0}$, let $\varepsilon>0, \delta>0$ be such that $M(\xi)<\frac{\varepsilon}{2}$.

Since $M$ is bounded Orlicz function, there exist an integer $G$ such that $M(X)<\frac{G}{2}$ for all $x \geq 0$.

Note that, for each $m, n, t \in N$

$$
\begin{aligned}
& \frac{1}{m n t} \sum_{i=1}^{m} \sum_{j=1}^{n} \sum_{k=1}^{t} M\left(\frac{D\left(x_{i j k}, x_{0}\right)}{\rho}\right) \\
= & \frac{1}{m n t} \sum_{i=1}^{m} \sum_{j=1}^{n} \sum_{k=1, D\left(x_{j i k}, x_{0}\right) \geq \varepsilon}^{t} M\left(\frac{D\left(x_{i j k}, x_{0}\right)}{\rho}\right) \\
& +\frac{1}{m n t} \sum_{i=1}^{m} \sum_{j=1}^{n} \sum_{k=1, D\left(x_{i j k}, x_{0}\right)<\varepsilon}^{t} M\left(\frac{D\left(x_{i j k}, x_{0}\right)}{\rho}\right) \\
\leq & M(\xi)+\frac{1}{m n t} \sum_{i=1}^{m} \sum_{j=1}^{n} \sum_{k=1, D\left(x_{i j k}, x_{0}\right) \geq \varepsilon}^{t} M\left(\frac{D\left(x_{i j k}, x_{0}\right)}{\rho}\right) \\
\leq & \frac{\varepsilon}{2}+\frac{G}{2}\left\{\frac{1}{m n t}\left|\left\{(i, j, k): D\left(x_{i j k}, x_{p q r}\right) \geq \xi, i \leq m, j \leq n, k \leq t\right\}\right| \geq \delta\right\} \\
\leq & \varepsilon+G\left\{\frac{1}{m n t}\left|\left\{(i, j, k): D\left(x_{i j k}, x_{p q r}\right) \geq \xi, i \leq m, j \leq n, k \leq t\right\}\right| \geq \delta\right\} \in I .
\end{aligned}
$$

Hence 


$$
\left\{(m, n, t) \in N \times N \times N: \frac{1}{m n t} \sum_{i=1}^{m} \sum_{j=1}^{n} \sum_{k=1}^{t} M\left(\frac{D\left(x_{i j k}, x_{0}\right)}{\rho}\right) \geq \delta\right\} \in I .
$$

Corollary 3.3. Let $x=\left\{x_{i j k}\right\}$ be a bound triple sequence of fuzzy number. Then $x$ is ideal statistically pre-Cauchy if and only if

$$
\left\{(m, n, t) \in N \times N \times N: \frac{1}{m^{2} n^{2} t^{2}} \sum_{i=1}^{m} \sum_{j=1}^{n} \sum_{k=1}^{t} D\left(x_{i j k}, x_{p q r}\right) \geq \delta\right\} \in I .
$$

Proof. Let $K=\sup _{i, j, k} D\left(x_{i j k}, \overline{0}\right)$ and defined $M(x)=\frac{(1+2 K) x}{1+x}$, then

$$
\begin{aligned}
& M\left(\frac{D\left(x_{i j k}, x_{p q r}\right)}{\rho}\right) \leq(1+2 K) D\left(x_{i j k}, x_{p q r}\right), \text { and } \\
& M\left(\frac{D\left(x_{i j k}, x_{p q r}\right)}{\rho}\right)=(1+2 K) \frac{D\left(x_{i j k}, x_{p q r}\right)}{1+D\left(x_{i j k}, x_{p q r}\right)} \\
& \geq \frac{\frac{(1+2 K) D\left(x_{i j k}, x_{p q r}\right)}{\rho}}{1+D\left(x_{i j k}, x_{p q r}\right)} \\
& \geq \frac{(1+2 K) D\left(x_{i j k}, x_{p q r}\right)}{1+2 A}=D\left(x_{i j k}, x_{p q r}\right) .
\end{aligned}
$$

Hence $\left\{(m, n, t) \in N \times N \times N: \frac{1}{m^{2} n^{2} t^{2}} \sum_{i=1}^{m} \sum_{j=1}^{n} \sum_{k=1}^{t} D\left(x_{i j k}, x_{p q r}\right) \geq \delta\right\} \in I$, if and only if $\left\{(m, n, t) \in N \times N \times N: \frac{1}{m^{2} n^{2} t^{2}} \sum_{i, p \leq m} \sum_{j, k \leq n} \sum_{t, r \leq t} M\left(\frac{D\left(x_{i j k}, x_{p q r}\right)}{\rho}\right) \geq \delta\right\} \in I$, and an immediate application of Theorem 3.1 completes the proof.

Corollary 3.4. Let $x=\left\{x_{i j k}\right\}$ be a bound triple sequence of fuzzy number. Then $x$ is ideal statistically convergent $x_{0}$ if and only if

$$
\left\{(m, n, t) \in N \times N \times N: \frac{1}{m n t} \sum_{i=1}^{m} \sum_{j=1}^{n} \sum_{k=1}^{t} D\left(x_{i j k}, x_{0}\right) \geq \delta\right\} \in I .
$$

Proof. Let $K=\sup _{i, j, k} D\left(x_{i j k}, \overline{0}\right)$ and defined $M(x)=\frac{\left(1+K+x_{0}\right) x}{1+x}$, then

$$
\begin{aligned}
M\left(\frac{D\left(x_{i j k}, x_{0}\right)}{\rho}\right) \leq\left(1+K+x_{0}\right) & D\left(x_{i j k}, x_{0}\right) \text {, and } \\
M\left(\frac{D\left(x_{i j k}, x_{0}\right)}{\rho}\right) & =\left(1+K+x_{0}\right) \frac{D\left(x_{i j k}, x_{0}\right)}{1+D\left(x_{i j k}, x_{0}\right)} \\
& \geq \frac{\left(1+K+x_{0}\right) D\left(x_{i j k}, x_{0}\right)}{\rho} \\
& \geq \frac{\left(1+K+x_{0}\right) D\left(x_{i j k}, x_{0}\right)}{1+K+x_{0}}=D\left(x_{i j k}, x_{0}\right) .
\end{aligned}
$$


Hence $\left\{(m, n, t) \in N \times N \times N: \frac{1}{m n t} \sum_{i=1}^{m} \sum_{j=1}^{n} \sum_{k=1}^{t} D\left(x_{i j k}, x_{0}\right) \geq \delta\right\} \in I$, if and only if $\left\{(m, n, t) \in N \times N \times N: \frac{1}{m^{2} n^{2} t^{2}} \sum_{i=1}^{m} \sum_{j=1}^{n} \sum_{k=1}^{t} M\left(\frac{D\left(x_{i j k}, x_{0}\right)}{\rho}\right) \geq \delta\right\} \in I$, and an immediate application of Theorem 3.1 completes the proof.

\section{Conclusion}

In this article, we introduced ideal statistically pre-Cauchy triple sequences of fuzzy numbers about Orlicz function. At the same time, we have proved some properties and relationships.

\section{Fund}

This work is supported by National Natural Science Fund of China (11761056); the Natural Science Foundation of Qinghai Province (2020-ZJ-920); University level planning project of Qinghai Minzu University (2021XJGH24).

\section{Conflicts of Interest}

The author declares no conflicts of interest regarding the publication of this paper.

\section{References}

[1] Fast, H. (1951) Sur la convergence statistique. Colloquium Mathematicum, 2, 241244. https://doi.org/10.4064/cm-2-3-4-241-244

[2] Buck, R.C. (1953) Generalized Asymptote Density. American Journal of Mathematics, 75, 335-346. https://doi.org/10.2307/2372456

[3] Schoenberg, I.J. (1959) The Integrability of Certain Functions and Related Summability Methods. The American Mathematical Monthly, 66, 361-375. https://doi.org/10.2307/2308747

[4] Altinok, H. and Et, M. (2015) On $\lambda$-Statistical Boundedness of Order $\beta$ of Sequences of Fuzzy Numbers. Soft Computing, 19, 2095-2100. https://doi.org/10.1007/s00500-015-1660-2

[5] Connor, J.S. (1988) The Statistical and Strong p-Cesàro Convergence of Sequences. Analysis, 8, 47-63. https://doi.org/10.1524/anly.1988.8.12.47

[6] Et, M. (2013) Generalized Cesàro Difference Sequence Spaces of Non-Absolute Type Involving Lacunary Sequences. Applied Mathematics and Computation, 219, 9372-9376. https://doi.org/10.1016/j.amc.2013.03.039

[7] Et, M., Altinok, H. and Altin, Y. (2004) On Some Generalized Sequence Spaces. Applied Mathematics and Computation, 154, 167-173. https://doi.org/10.1016/S0096-3003(03)00700-8

[8] Et, M. and Sengül, H. (2014) Some Cesàro-Type Summability Spaces of Order $\alpha$ and Lacunary Statistical Convergence of Order $\alpha$. Filomat, 28, 1593-1602. https://doi.org/10.2298/FIL1408593E

[9] Fridy, J. (1985) On Statistical Convergence. Analysis, 5, 301-313. https://doi.org/10.1524/anly.1985.5.4.301 
[10] Fridy, J. and Orhan, C. (1993) Lacunary Statistical Convergence. Pacific Journal of Mathematics, 160, 43-51. https://doi.org/10.2140/pjm.1993.160.43

[11] Mursaleen, M. (2000) $\lambda$-Statistical Convergence. Math Slovaca, 50, 28-37.

[12] Matloka, M. (1986) Sequences of Fuzzy Numbers. BUSEFAL, 28, 28-37.

[13] Nuray, F. and Savas, E. (1995) Statistical Convergence of Fuzzy Real Numbers. Mathematica Slovaca, 45, 269-273.

[14] Tripathy, B.C. and Baruah, A. (2010) Lacunary Statistically Convergent and Lacunary Strongly Convergent Generalized Difference Sequences of Fuzzy Real Numbers. Kyungpook Mathematical Journal, 50, 565-574. https://doi.org/10.5666/KMJ.2010.50.4.565

[15] Canak, I. (2014) Tauberian Theorems for Cesàro Summability of Sequences of Fuzzy Numbers. Journal of Intelligent \& Fuzzy Systems, 27, 937-942. https://doi.org/10.3233/IFS-131053

[16] Mursaleen, M. and Mohiuddine, S.A. (2009) On Lacunary Statistical Convergence with Respect to the Intuitionistic Fuzzy Normed Space. Journal of Computational and Applied Mathematics, 233, 142-149. https://doi.org/10.1016/j.cam.2009.07.005

[17] Savas, E. (2000) A Note on Sequence of Fuzzy Numbers. Information Sciences, 124, 297-300. https://doi.org/10.1016/S0020-0255(99)00073-0

[18] Hazarika, B. (2013) Lacunary Difference Ideal Convergent Sequence Spaces of Fuzzy Numbers. Journal of Intelligent and Fuzzy Systems, 25, 157-166. https://doi.org/10.3233/IFS-2012-0622

[19] Aytar, S. and Pehlivan, S. (2008) Statistical Convergence of Sequences of Fuzzy Numbers and Sequences of $\alpha$-Cuts. International Journal of General Systems, 37, 231-237. https://doi.org/10.1080/03081070701251075

[20] Lindenstrauss, J. and Tzafiri, L. (1971) On Orlicz Sequence Spaces. Israel Journal of Mathematics, 10, 379-390. https://doi.org/10.1007/BF02771656

[21] Connor, J.S., Fridy, J. and Kline, J. (1994) Statistically Pre-Cauchy Sequences. Analysis, 14, 311-317. https://doi.org/10.1524/anly.1994.14.4.311

[22] Barlak, D. (2020) Statistical Convergence of Order $\beta$ for $(\lambda, \mu)$ Double Sequences of Fuzzy Numbers. Journal of Intelligent and Fuzzy Systems, 39, 6949-6954. https://doi.org/10.3233/JIFS-200039

[23] Saini, K., Raj, K. and Mursaleen M. (2021) Deferred Cesaro and Deferred Euler Equi-Statistical Convergence and Its Applications to Korovkin-Type Approximation Theorem. International Journal of General Systems, 50, 567-579. https://doi.org/10.1080/03081079.2021.1942867

[24] Nuray, F. and Savaş, E. (1998) Lacunary Statistical Convergence of Sequences of Fuzzy Numbers. Fuzzy Sets \& Systems, 99, 353-355. https://doi.org/10.1016/S0165-0114(98)00031-1

[25] Parashar, S.D. and Choudhary, B. (1994) Sequence Spaces Defined by Orlicz Functions. Indian Journal of Pure and Applied Mathematics, 25, 419-428.

[26] Tripathy, B.C. and Mehta, S. (2003) On a Class of Sequences Related to $l^{p}$ Space Defined by Orlicz Functions. Soochow Journal of Mathematics, 29, 379-391.

[27] Sahiner, A., Gürdal, M. and Düden, K. (2007) Triple Sequences and Their Statistical Convergence. Selcuk Journal of Applied Mathematics, 8, 49-55.

[28] Sabiha, T. (2018) Statistically Pre-Cauchy Triple Sequences and Orlicz Functions. Pure Mathematical Sciences, 7, 1-9. https://doi.org/10.12988/pms.2018.756 